\title{
JAXA-NASA Interoperability Demonstration for Application of DTN under Simulated Rain Attenuation
}

\author{
Kiyohisa Suzuki ${ }^{1}$, Shinichi Inagawa ${ }^{2}$ \\ Japan Aerospace Exploration Agency, Tsukuba, Ibaraki, 305-8505, Japan \\ and \\ Jeff Lippincott ${ }^{3}$, Andrew J. Cecil ${ }^{4}$ \\ National Aeronautics and Space Administration, Huntsville, Alabama, 35812, United States of America
}

\begin{abstract}
As is well known, K-band or higher band communications in space link segment often experience intermittent disruptions caused by heavy rainfall. In view of keeping data integrity and establishing autonomous operations under such situation, it is important to consider introducing a tolerance mechanism such as Delay/Disruption Tolerant Networking (DTN). The Consultative Committee for Space Data Systems (CCSDS) is studying DTN as part of the standardization activities for space data systems. As a contribution to CCSDS and a feasibility study for future utilization of DTN, Japan Aerospace Exploration Agency (JAXA) and National Aeronautics and Space Administration (NASA) conducted an interoperability demonstration for confirming its tolerance mechanism and capability of automatic operation using Data Relay Test Satellite (DRTS) space link and its ground terminals. Both parties used the Interplanetary Overlay Network (ION) open source software, including the Bundle Protocol, the Licklider Transmission Protocol, and Contact Graph Routing. This paper introduces the contents of the interoperability demonstration and its results.
\end{abstract}

\section{Introduction}

$\mathrm{T}$

he Delay/Disruption Tolerant Networking (DTN) protocols are convincing candidate protocols for higher band space communications such as K-band and optics. Those communications often experience intermittent disruptions caused by the effect of rainfall or clouds between the space segment and the Earth ground segment. From a standpoint of maintaining data integrity and establishing autonomous operations under such situations, it is important to consider introducing a tolerance mechanism.

The Consultative Committee for Space Data Systems (CCSDS) has studied Solar System Internet (SSI) architecture and DTN standards as a space communication protocol standard since 2008.[1][2] As the name suggests, DTN has the ability to handle both short interruptions of communication (disruption) and longer interruptions of communication (delay). DTN is an overlay network using the communication medium available. This enables DTN to be a part of the core protocol in SSI.

As a contribution to CCSDS and a feasibility study for future utilization of DTN, JAXA and NASA performed an interoperability demonstration to confirm its tolerance mechanism and capability of automatic operation used by JAXA's Data Relay Test Satellite (DRTS) space link and its ground terminals. To demonstrate and checkout the capabilities test cases included actual space link delay, simulated rain attenuation, and simulated long haul delay.

The purpose of this paper is to overview DRTS testing and findings from the experiments performed.

\footnotetext{
${ }^{1}$ Engineer ,Consolidated Space Track and Data Acquisition Department, 2-1-1 Sengen, Tsukuba, Ibaraki, Japan

${ }^{2}$ Engineer, Consolidated Space Track and Data Acquisition Department, 2-1-1 Sengen, Tsukuba, Ibaraki, Japan

${ }^{3}$ Software Engineer, Mission Operations Systems Branch, jeff.lippincott@nasa.gov, EO50, Huntsville, AL 35812

${ }^{4}$ Systems Engineer, Mission Operations Systems Branch, andrew.j.cecil@nasa.gov, EO50, Huntsville, AL 35812
} 


\section{Facilities and equipment for DRTS testing}

\section{A. Overview}

To perform DRTS testing, JAXA's Space Network was used. Communication with DRTS requires other portions of the CCSDS protocol stack for connectivity. This required some additional equipment to implement the test.

Figure 1 illustrates an overview of the test configuration for DRTS testing.

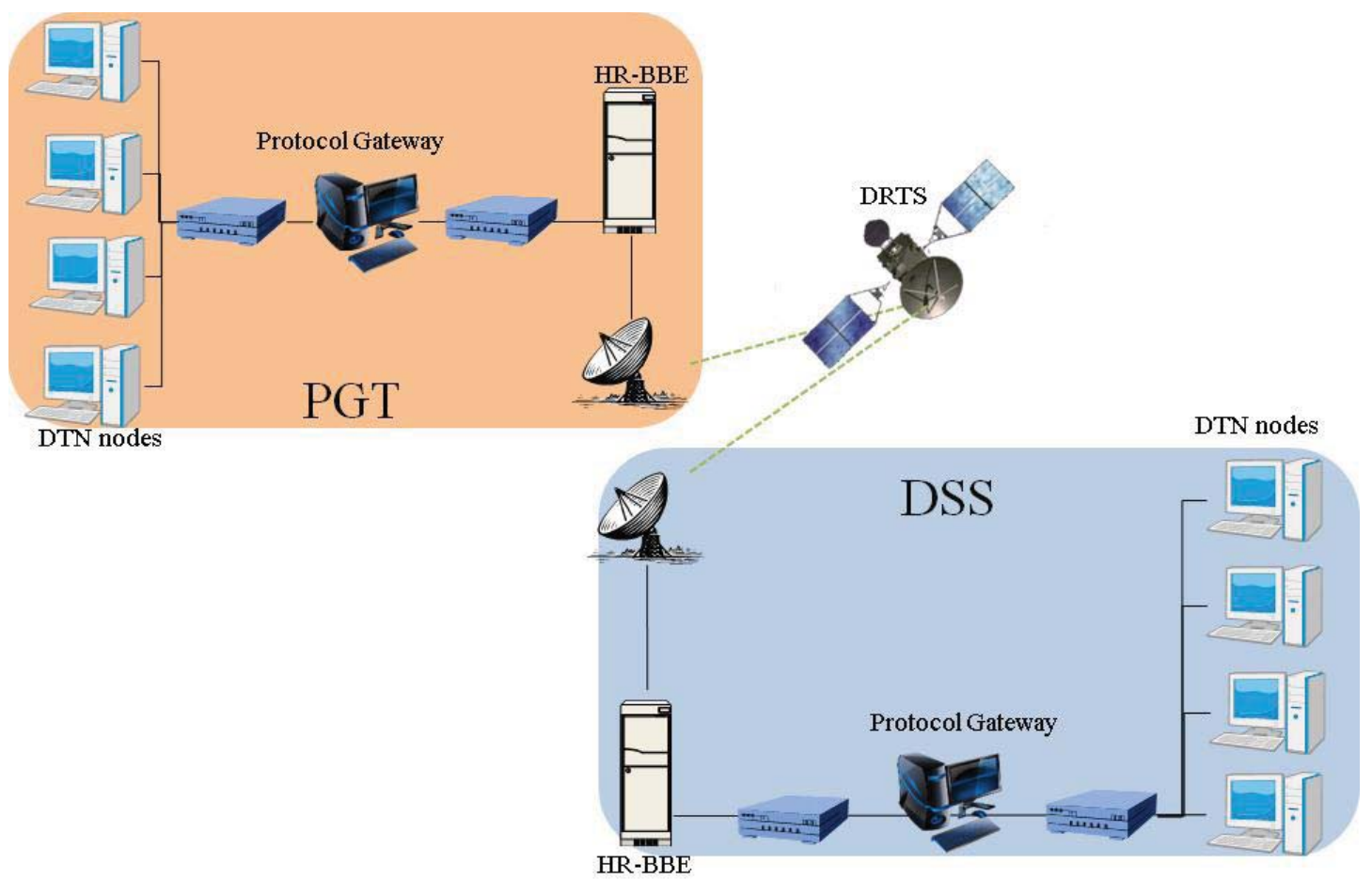

Figure 1. Overview of the test configuration for DRTS testing

Figure 2 illustrates the test configuration connectivity.

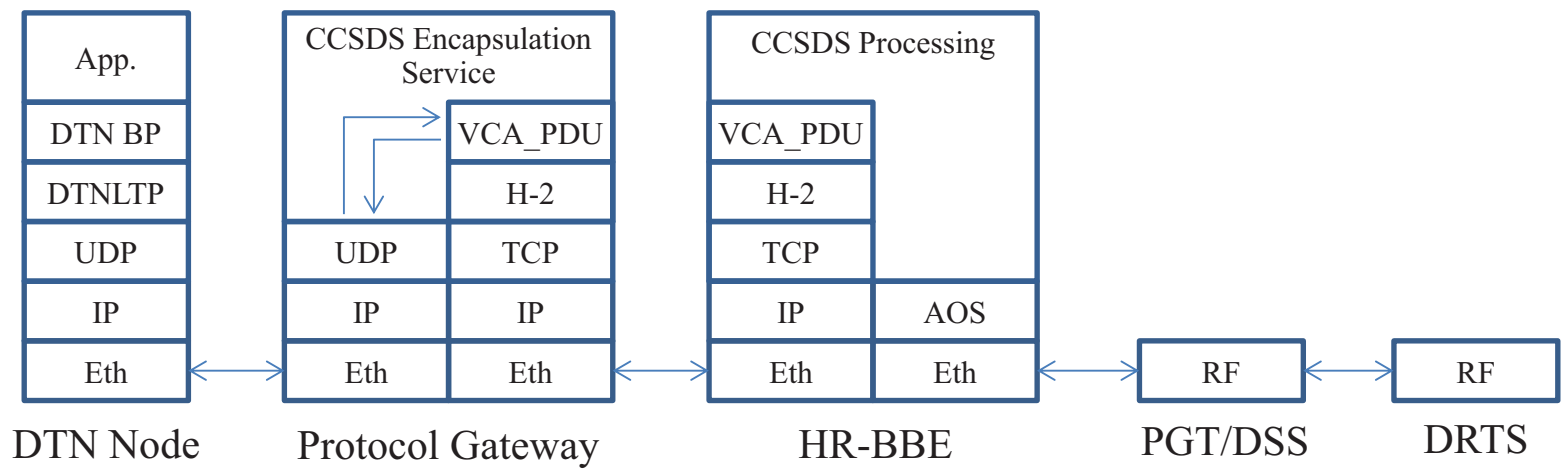

Figure 2. The connectivity of the test configuration

\section{B. DTN Implementation}

To perform testing, the Interplanetary Overlay Network (ION) version 3.1.3 open source software implementation of DTN (http://sourceforge.net/projects/ion-dtn/) was used on Linux PCs. ION can be executed within a Local Area Network (LAN) environment. The following components in ION were used for preparations and testing. 
The Bundle Protocol: The Bundle Protocol (DTN BP) is implemented based on RFC 5050 [3] and is necessary to support the DTN in space applications. It represents an overlay implementation that is extensible to many link layer protocols including the Internet Protocol. It will support the interoperability between ground and space based operations and is resilient to the harsh environment which characterizes spacecraft operations from low Earth to Deep Space.

The Licklider Transmission Protocol: The Licklider Transmission Protocol (DTN LTP) provides optional reliability mechanisms on top of an underlying (usually data link) communication service such as TCP without session. DTN LTP is based on RFC 5326 [4]. When LTP is operating underneath DTN BP, the convergence layer adapter mediating the two will be responsible for translating between DTN endpoint IDs and LTP engine IDs in an implementation-specific manner.

Contact Graph Routing: Contact Graph Routing (CGR) is a candidate routing algorithm for DTN communication. [5] CCSDS continues studies to standardize this algorithm for SSI. CGR determines communication paths dynamically based on the visibilities of neighbor nodes.

For execution of CGR, a Contact Plan is required. A Contact Plan is list of contacts where each contact is defined by a visibility (communication path) timeline, distance, and bandwidth between own node and a neighbor node. DTN enables automatic data transfer between any nodes on the network by this simple definition.

\section{JAXA Space Network}

JAXA Space Network is formally called the "DRTS Space Network Experiment System (DRTS SN)." The system was developed for the purpose of verifying inter-satellite communication technology using JAXA's geostationary (GEO) data relay satellite, DRTS. DRTS was launched from Tanegashima Space Center in September 2002, and placed into GEO in October 2002. DRTS has two communication links. One is Inter Orbit Link (IOL) which provides communications between DRTS and user spacecraft. Another is Feeder link (FL) which provides communications between DRTS and ground terminals.6]

The Primary Ground Terminal (PGT) and DRTS System-calibration Station (DSS) were used as end points for the RF communication link during testing. PGT is the main ground terminal for JAXA space network at Tsukuba Space Center (TKSC). DSS is a ground terminal for valuable RF simulation with user spacecraft and also a calibration ground terminal for Space Network at TKSC.

Both ground terminals were included on High Rate-Base Band Equipment (HR-BBE) where data was converted between the CCSDS AOS Space Data Link Protocol [7] for ground to space communication and IP traffic for ground to ground communication.

Figure 3 illustrates DRTS on-orbit configuration.

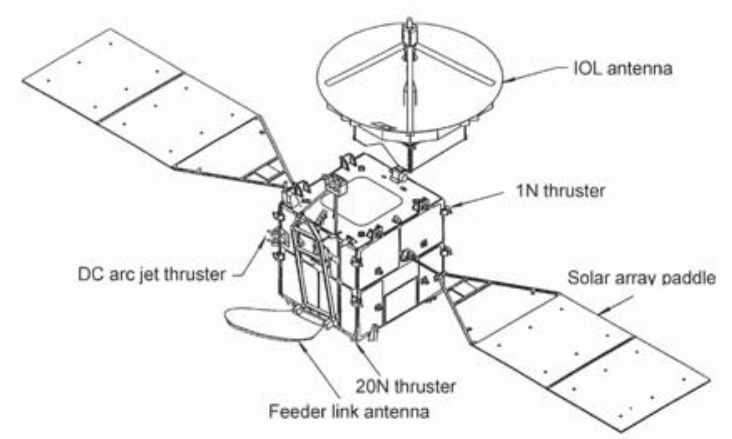

Figure 3. DRTS on-orbit configuration.

\section{Delay simulator}

In general, delay simulators in the LAN environment perform within tens of seconds per Round Trip Time (RTT). From the standpoint of our objective, we needed to insert tens of minutes per RTT corresponding to the delay between Earth and Mars. Therefore, JAXA implemented the delay simulator which could meet our needs. The delay simulator was implemented on a Linux PC and used multiple virtual Operating Systems (OS). 
Figure 4 illustrates a conceptual diagram delay simulator applying 600 second delay per RTT.

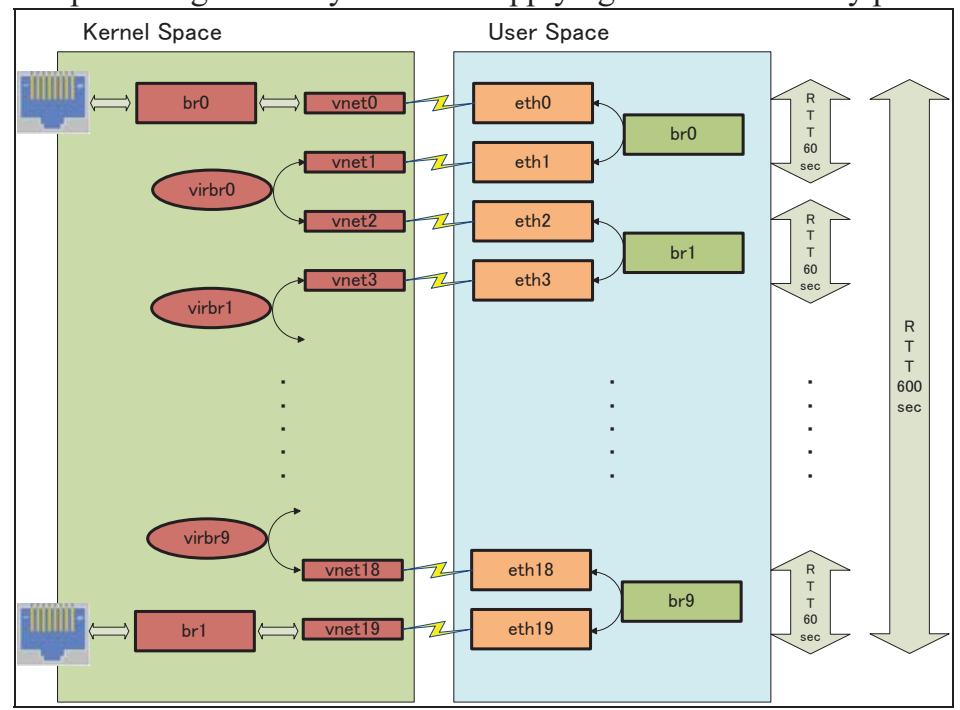

Figure 4. Conceptual Diagram of delay simulator.

As a result of implementation, this simulator achieved a maximum delay of 3,600 seconds per RTT. Figure 5 illustrates the results of delay condition.

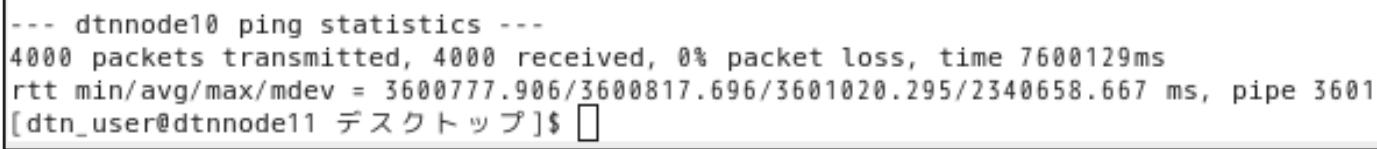

Figure 5. The results of delay condition.

\section{E. Protocol Gateway}

The protocol data length for UDP is variable up to $64 \mathrm{kB}$. In contrast, the protocol data length for a CCSDS transfer frame is fixed around $1 \mathrm{kB}$. Fragmentation and re-assembling of UDP packets is occurring. Based on that fact, the protocol gateway (see Figure 2) was implemented:

1) To absorb the difference of protocol interface between HR-BBE and DTN node.

2) To apply the CCSDS Encapsulation Service [8] as a method handling fragmentation and re-assembling.

Figure 6 illustrates the mechanism of protocol gateway.

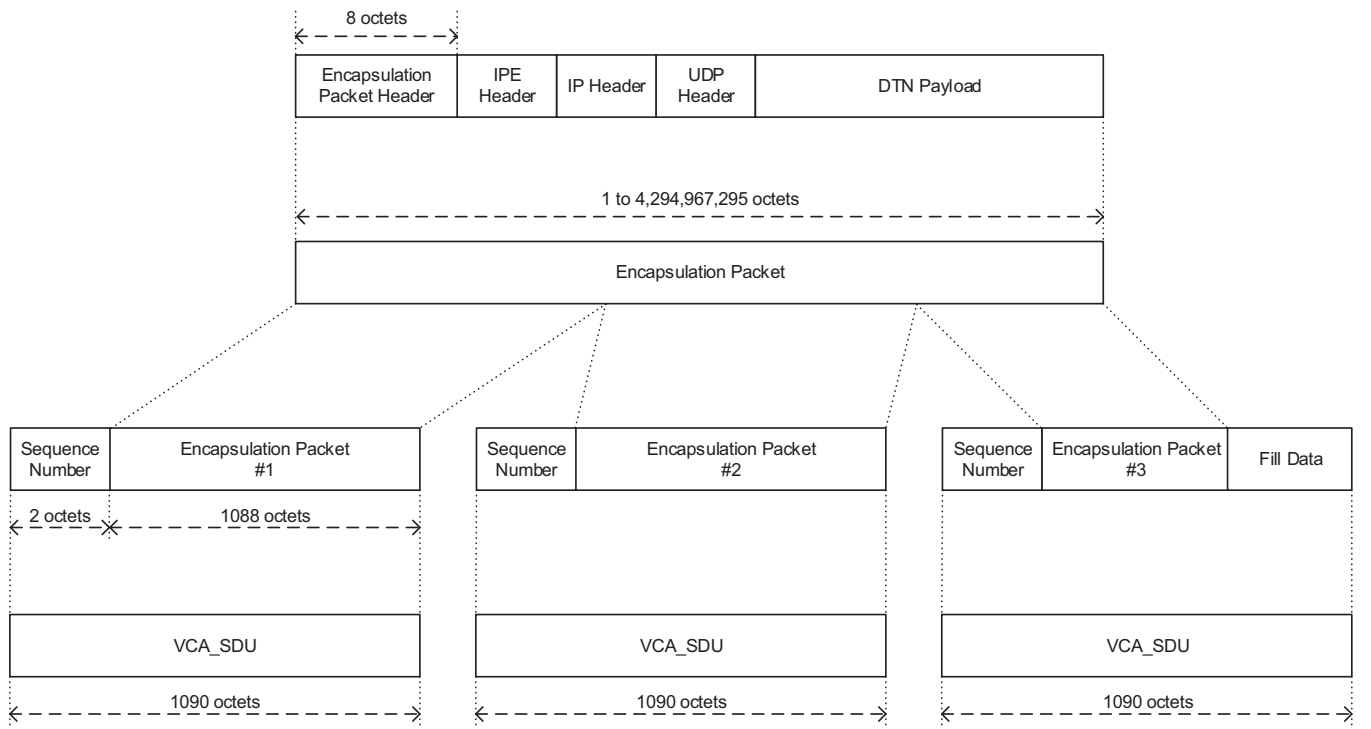

Figure 6. The mechanism of protocol gateway. 


\section{Classifications and definitions for delay and disruption}

Classifications and definitions for delay and disruption were important to clarify test conditions and to value DTN's feasibility from the results. We studied classifications and definitions mainly from the standpoint of spacecraft operation.

\section{A. Delay}

Preparation on the ground: The delay simulator was used, if the required delay for a test item was greater than the actual delay in internet environment.

DRTS testing: An actual propagation delay and processing delay between DRTS (GEO satellite) and two ground terminals were basically embedded. The delay simulator was used, if the required delay for a test item was greater than the actual delay in the test configuration.

\section{B. Disruption}

Predictable disruption: A predictable disruption was defined as a known loss of sight between spacecraft. This type of disruption was set in the contact plan on each DTN node. Therefore, the expected behavior was that each DTN node could cognize predictable disruptions, and did not forward bundles during predictable disruptions. Predictable disruption was simulated by disconnection of the RF link.

Unpredictable disruption: An unpredictable disruption was defined as an unplanned, intermittent loss of communication caused by radio interference and/or heavy rain attenuation. Unpredictable disruption could not be set in the contact plan on each DTN node. Therefore, the expected behavior was that each DTN node could not cognize unpredictable disruptions and DTN node continued re-forwarding bundles until a link is re-established during the contact. After the contact expires, the DTN node would then search for a new route to continue forwarding bundles towards the destination DTN node. Unpredictable disruption was simulated by disconnection of the RF link.

Simulated rain attenuation process: The rain attenuation process was defined as manipulating the RF level (either reducing or increasing) to mimic the attenuation levels from actual rain.

\section{Preliminary testing}

Before DRTS testing, we performed interoperability ground testing between each agency site through the internet. The earlier preliminary testing of both parties was shown [9]. The objective of this testing was to optimize the test plan and DTN parameters prior to DRTS testing.

The expected results for all test items were:

1) All bundles sent arrive at destination node. Monitoring provided by LAN analyzer.

2) The transferred file (an image file) to the destination nodes is completely the same at source node by comparing the hash value.

Figure 7 illustrates the configuration of this testing. And Table 1 illustrates summary of results. 


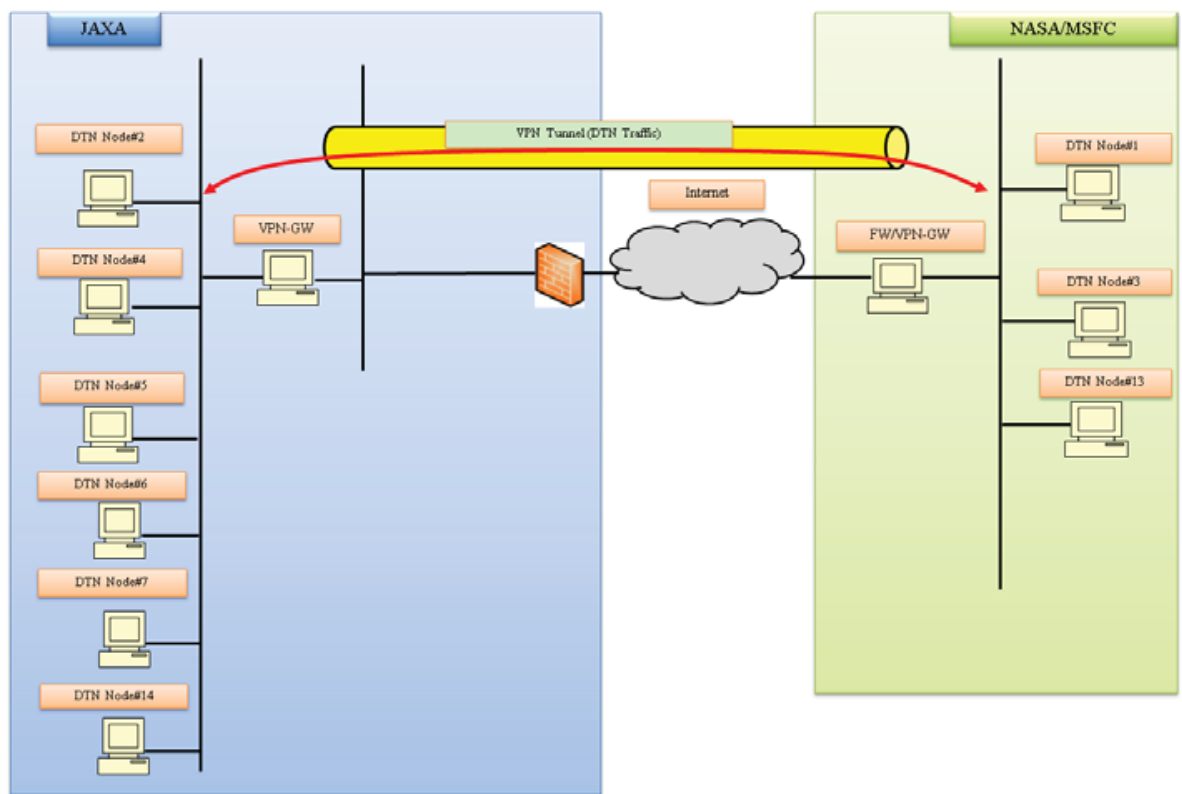

Figure 7. The configuration of interoperability ground testing.

Table 1. Summary of results

\begin{tabular}{|l|l|l|l|}
\hline No & Item & Result & Date \\
\hline 1 & DTN connectivity & OK & 10 June 2013 \\
\hline 2 & $\begin{array}{l}\text { File transfer by CFDP over DTN under delay and predictable } \\
\text { and unpredictable disruption. }\end{array}$ & OK & 11 June 2013 \\
\hline 3 & $\begin{array}{l}\text { File transfer by CFDP over DTN between four nodes under } \\
\text { delay and predictable and unpredictable disruption. }\end{array}$ & OK & 12 June 2013 \\
\hline 4 & $\begin{array}{l}\text { File transfer by CFDP over DTN between seven nodes under } \\
\text { delay and predictable and unpredictable disruption }\end{array}$ & OK & 13 June 2013 \\
\hline
\end{tabular}

We concluded that to perform DRTS testing was feasible.

\section{DRTS testing}

We performed DRTS testing in July 2013. The main items and results of DRTS testing were following.

\section{A. File transfer by CFDP over DTN with simulated rain attenuation process}

The purpose of this item was to confirm a feasibility of delay and disruption tolerance for DTN through a DTN implementation. For this test intermittent disruption from rain attenuation was simulated by shifting the balance of $\mathrm{C} / \mathrm{N}$ (Carrier to Noise ratio) input of HR-BBE on time axis. A noise generator was used to step up/down on time axis to simulate a behavior of rain attenuation. The expected results of this item were 1) bundles sent all arrive at destination node through space link and 2) the transferred file (an image file) to the destination nodes is completely the same at source node. The hash value was used as the method of comparing between the file at source node and the file at destination node.

Figure 8 and Figure 9 illustrates the topology and configuration.

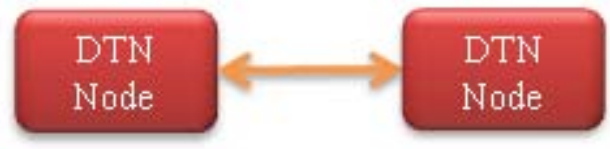

Figure 8 The topology 


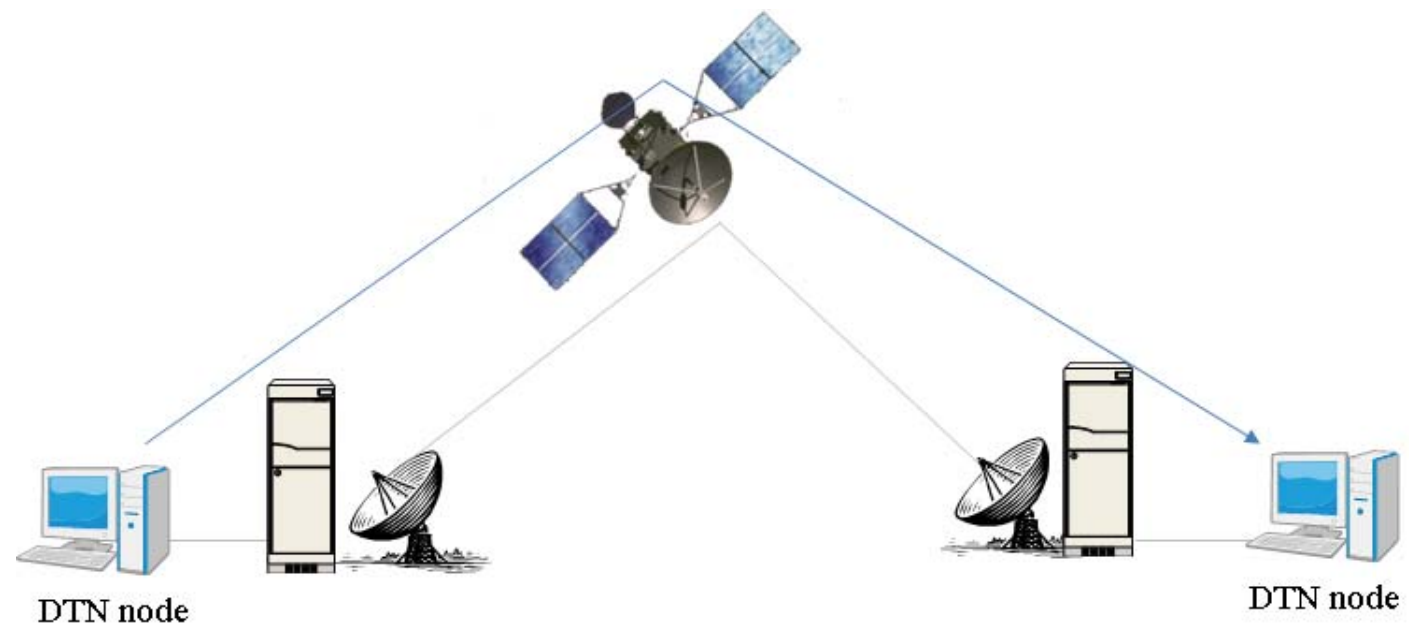

Figure 9. The test configuration

Test Result: The hash value between source and destination was confirmed to match. Figure 10 illustrates situation of file transfer.

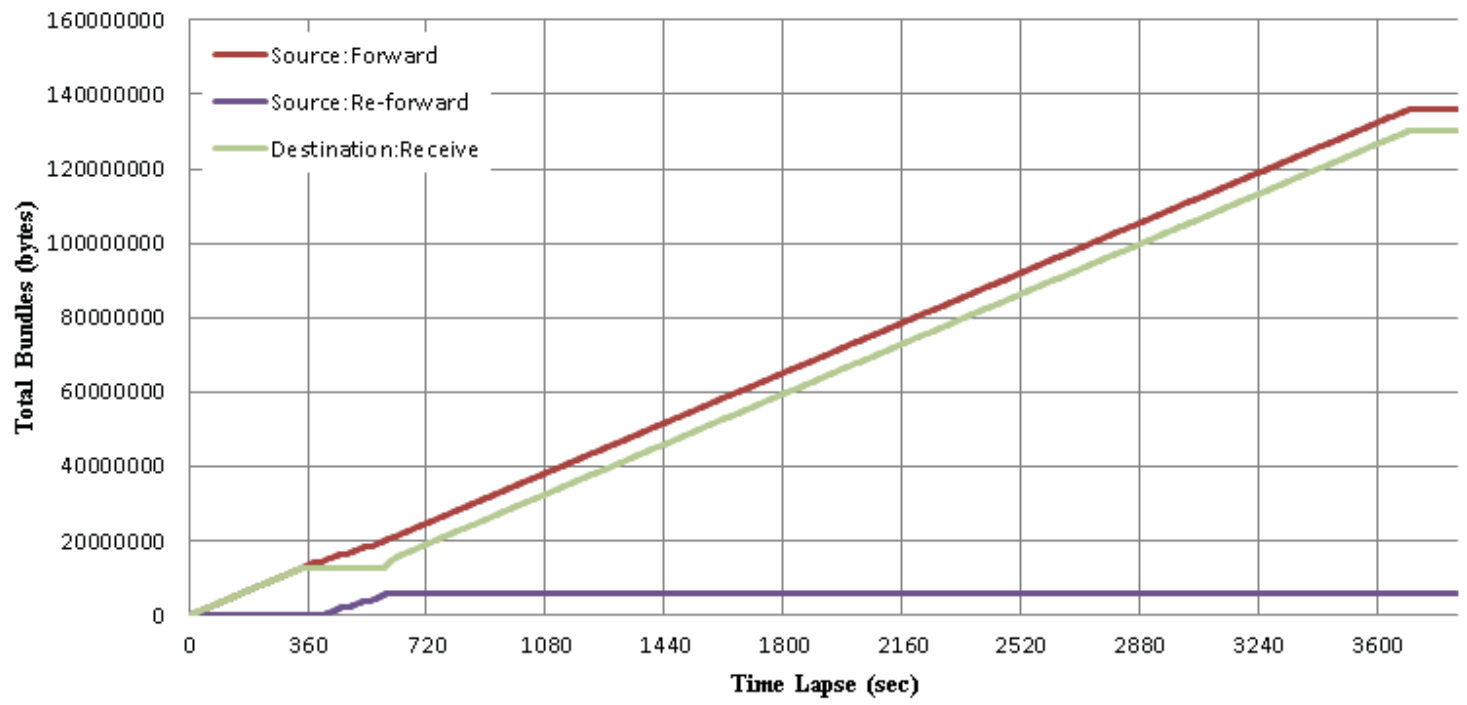

Figure 10. The situation of file transfer

If you look at the Figure 10, you will see that the bundle transmitted, re-transmitted, and was received at the destination node despite the occurrence of simulated rain attenuation between 240 and 720 seconds (the occurrence of disruption between 360 seconds and 600 seconds.). And we confirmed that the hash value completely matched. As the result of this item, we concluded that the following test objectives were confirmed for peer-to-peer configuration under delay from ground to ground via GEO relay satellite and impressing predictable/unpredictable disruption.

- Data transfer was automatically recovered and finished after intermittent disruptions in an actual space link.

- Integrity for a chunk of data such as an image file was maintained at destination.

\section{B. CGR between seven nodes under long haul delay and disruption.}

The purpose of this item was to confirm the feasibility of CGR under long haul delay and disruption through a DTN implementation. We assumed a test scenario for a multiple deep space relay communication to confirm feasibility. The topology was comprised of a single MOC, a single Rover on planet surface, Triple Relay Satellite (Planet 
orbiter and GEO relay), and two ground terminals for GEO relay. Also this simulated scenario was demonstrated by data transfer over a network where long disconnection and only gradual connection between several hops are expected. DTN is expected to be capable to automatically select a route from the contact plan even in such difficult network environment. DTN could also transfer the whole data to the destination node.

We defined each DTN node as follows,

Rover node: A rover was a probe vehicle on a planet surface such as Mars. In this test item, a rover was the source DTN node.

Relay satellite node: Relay satellites included GEO data relay satellite (GEO1) and Relay Orbiter around a Planet (ROP1, ROP2). GEO data relay satellite comprised a part of Space Network. Relay Orbiter around a Planet takes four hours per revolution. In this test item, all relay satellites were DTN nodes with the role of gateway for forwarding sensor data to the MOC. The RTT between ROP and GEO1 was set tol hour in the delay simulator.

Ground terminals for GEO relay node: Ground terminals for relay satellite were ground stations (GT1, GT2) which also comprise Space Network. In this test item, ground terminals were DTN nodes with the role of gateway between relay satellite and MOC. Normally, Space Network is operated by only one ground terminal called "Primary ground terminal" in addition to a backup terminal.

MOC node: A Mission Operation Centre was the destination DTN node.

Figure11 and Figure12 illustrates the topology and configuration.

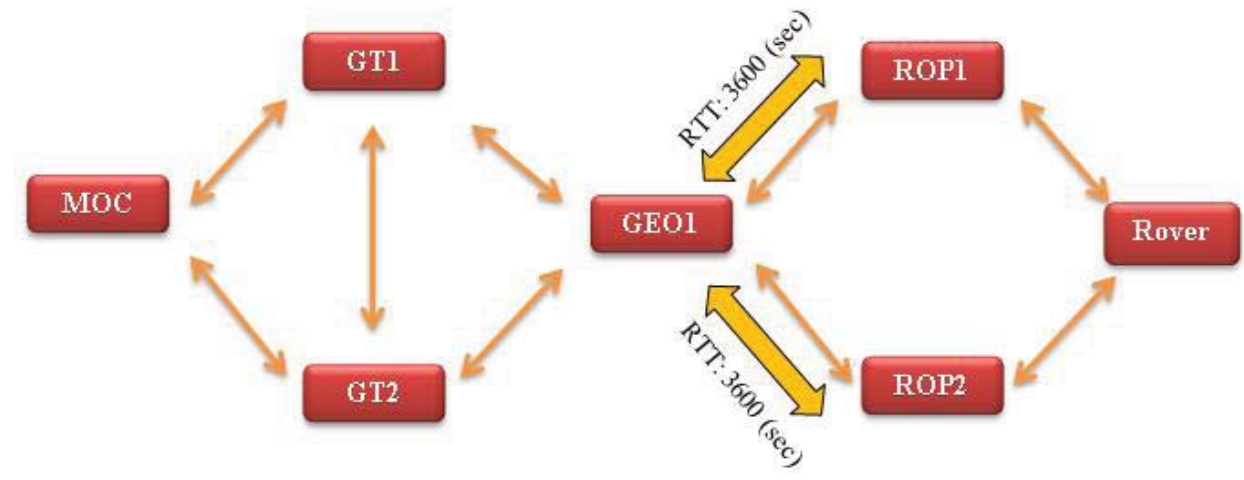

Figure 11. The topology

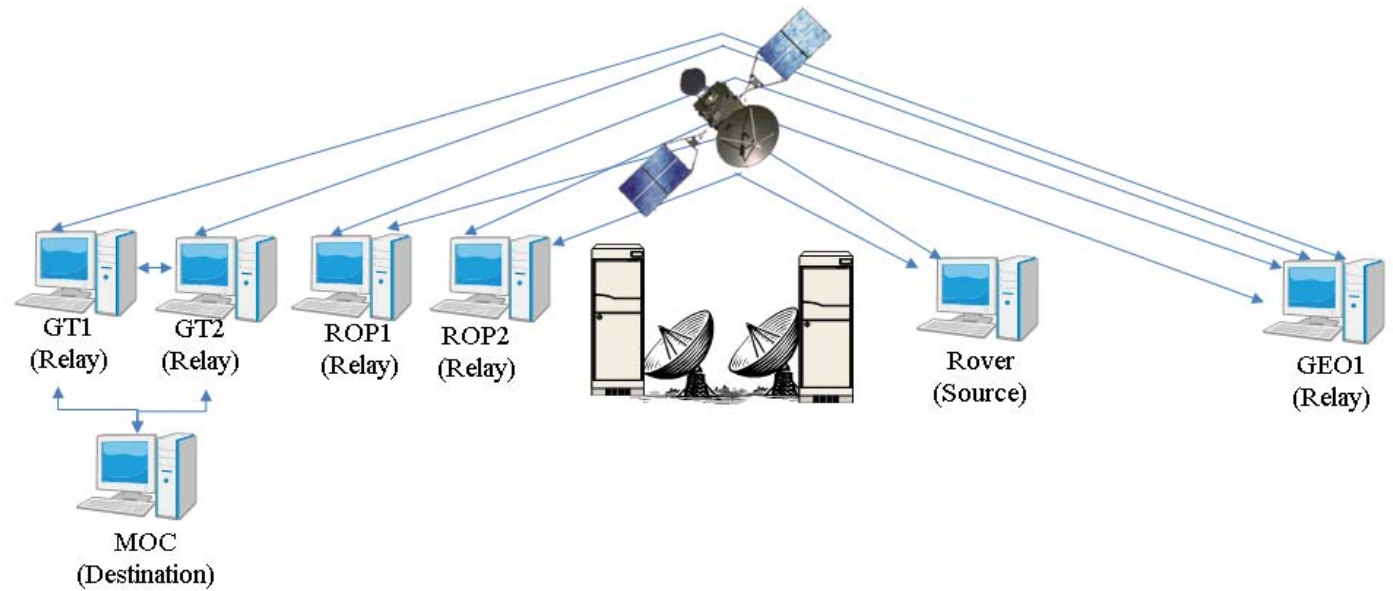

Figure 12. The test configuration 
Test Result: The hash value between the rover node and the MOC was confirmed to match. Figure 13, 14, 15, and 16 illustrate situation of file transfer. If you look at the Figure 13 and Figure 16, you will see that all of the bundles arrive at the destination node despite the occurrence of disruption, long-haul delay, and route selection.

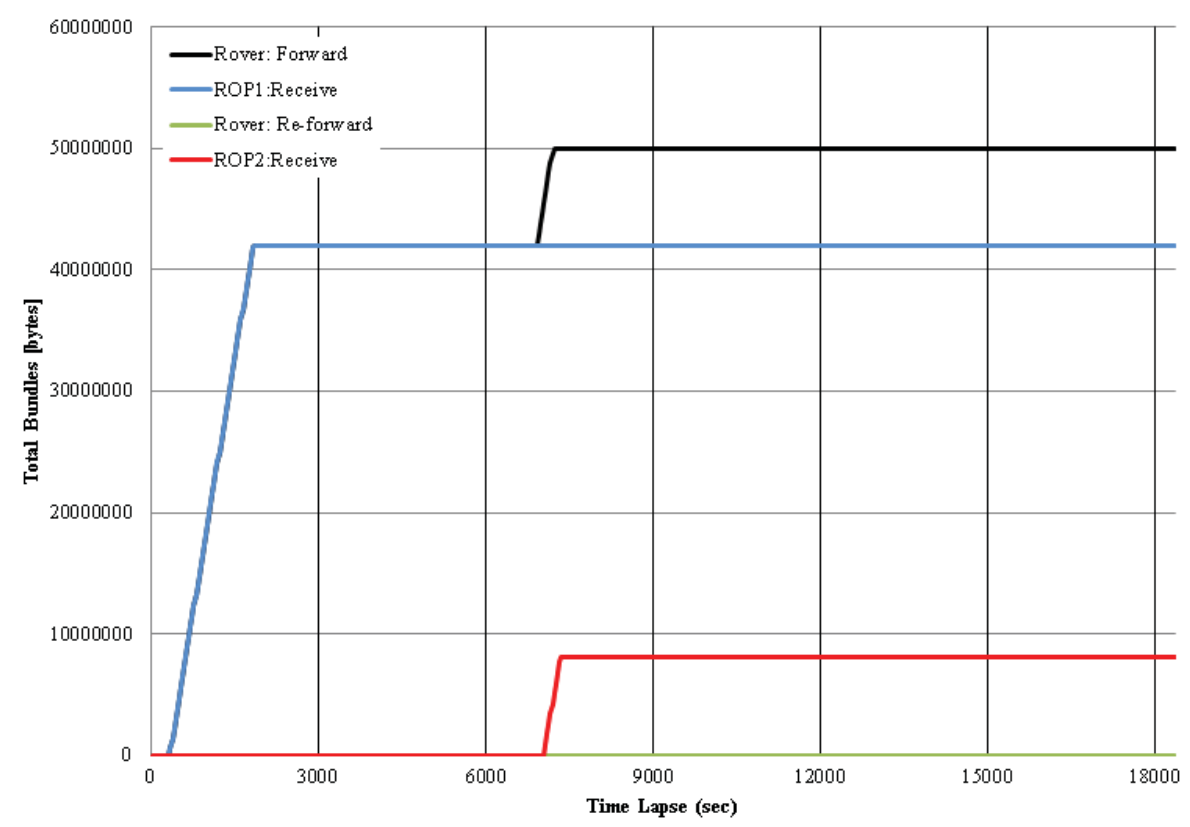

Figure 13. The situation of file transfer from Rover to ROP1/2

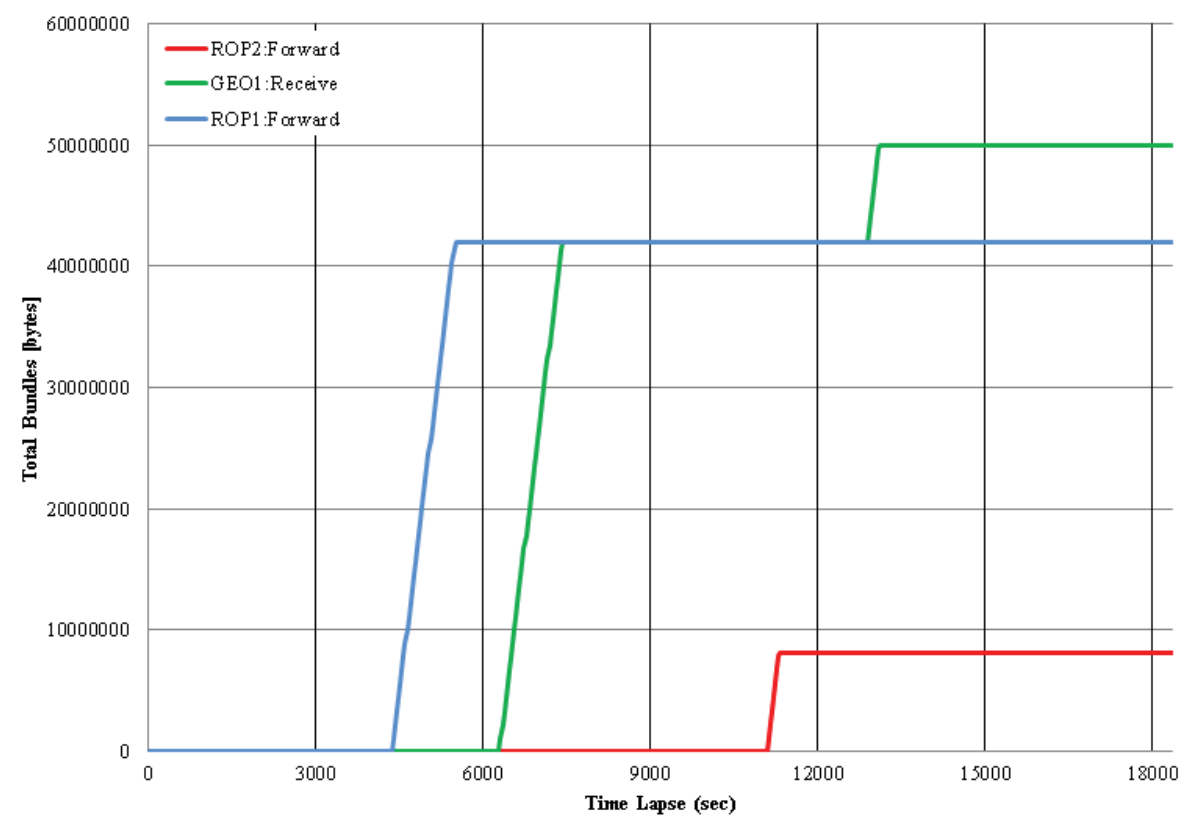

Figure 14. The situation of file transfer from ROP1/2 to GEO1 


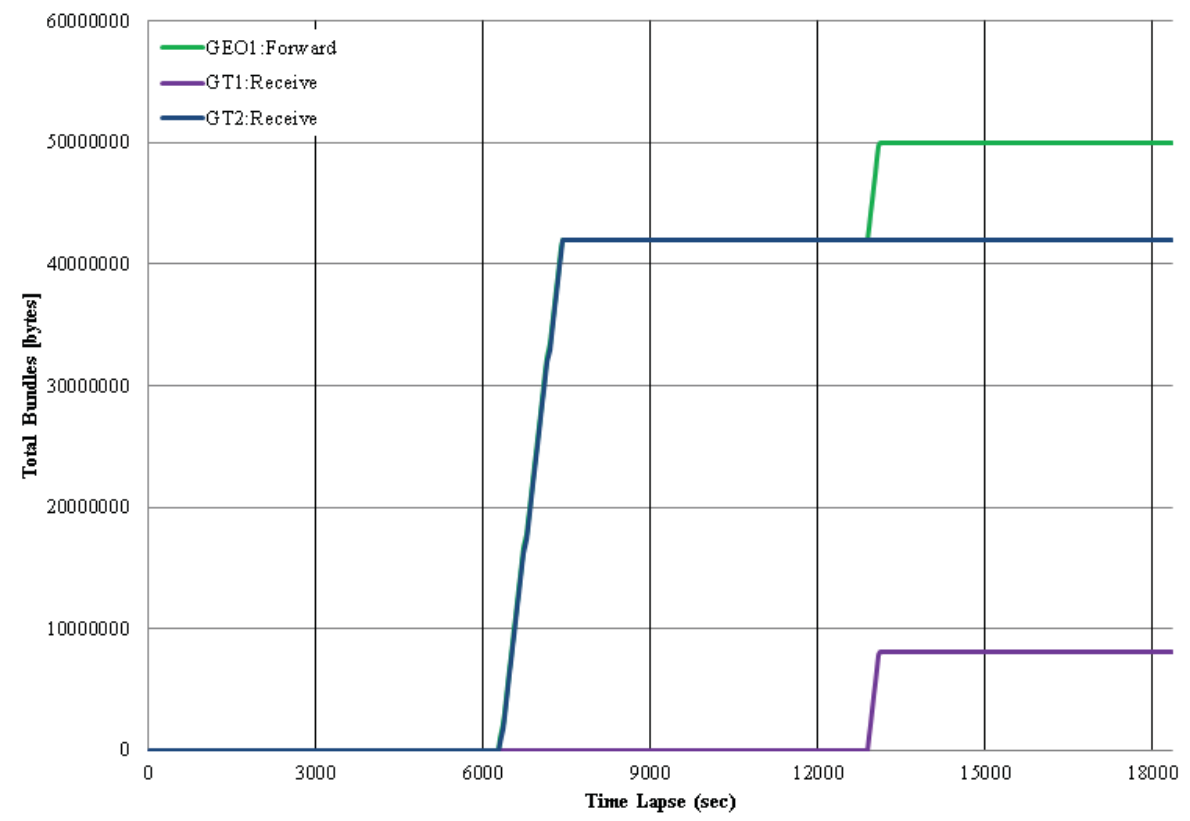

Figure 15. The situation of file transfer from GEO1 to GT1/2

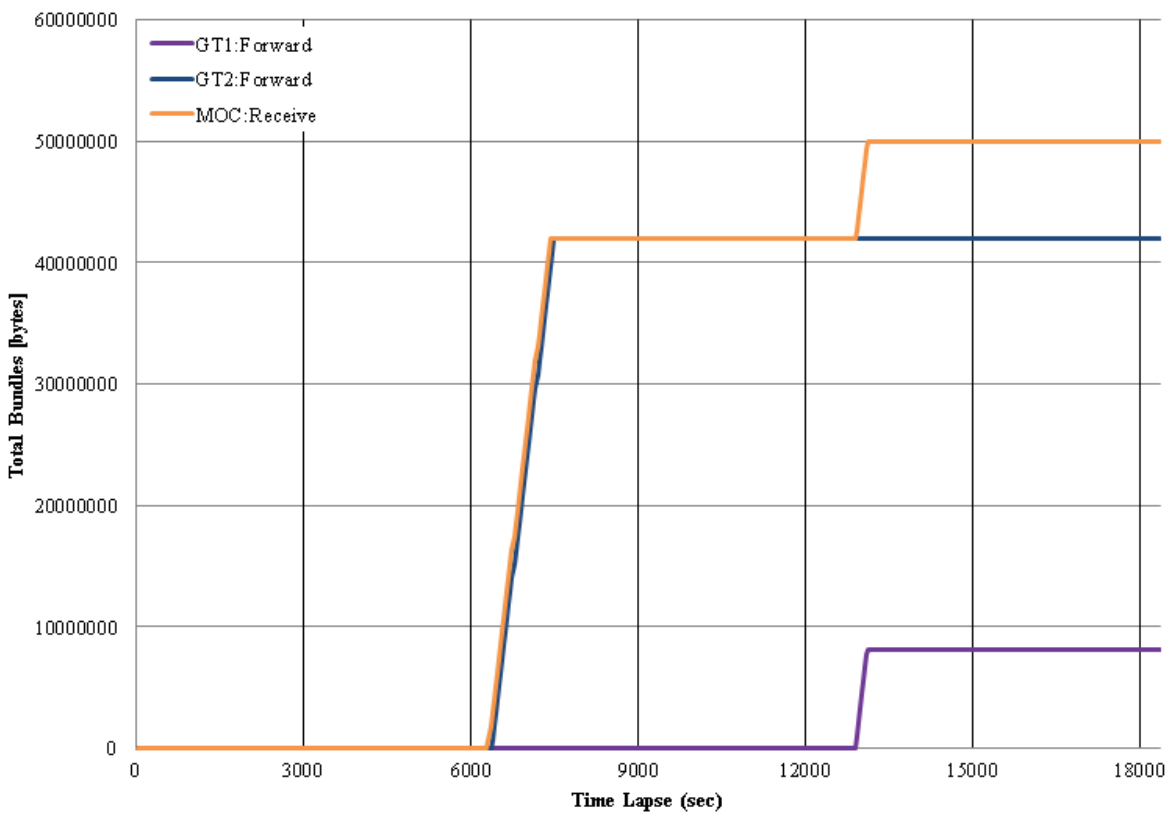

Figure 16. The situation of file transfer from GT1/2 to MOC

As the result of this test item, we concluded that the full of test objectives was confirmed for a complex topology under delay from ground to ground via GEO relay satellite.

- Data transfer was automatically recovered and finished after intermittent disruptions in an actual space link.

- Integrity for a chunk of data such as an image file was maintained at destination.

- Transfer routes are automatically selected in accordance with calculation under long haul delay and disruption. 


\section{Discussion}

The results of DRTS testing imply that DTN has feasibility for long haul delay and disruption tolerance in an actual space link environment.

\section{A. Comparison a performance between DTN/UDP/IP and TCP/IP under delay environment}

The first point to discuss is a comparison of performance between commodity internet and DTN as a kind of SSI protocol from the standpoint of delay. Establishment of related assets could be cheaper if commodity internet could apply for any space link segments.

We performed an additional feasibility study of delay tolerance by comparing DTN and TCP/IP. Specifically, we focused on the throughput of a file transfer using DTN and TCP/IP under varying time delays. Figure 17 illustrates the configuration of feasibility study.

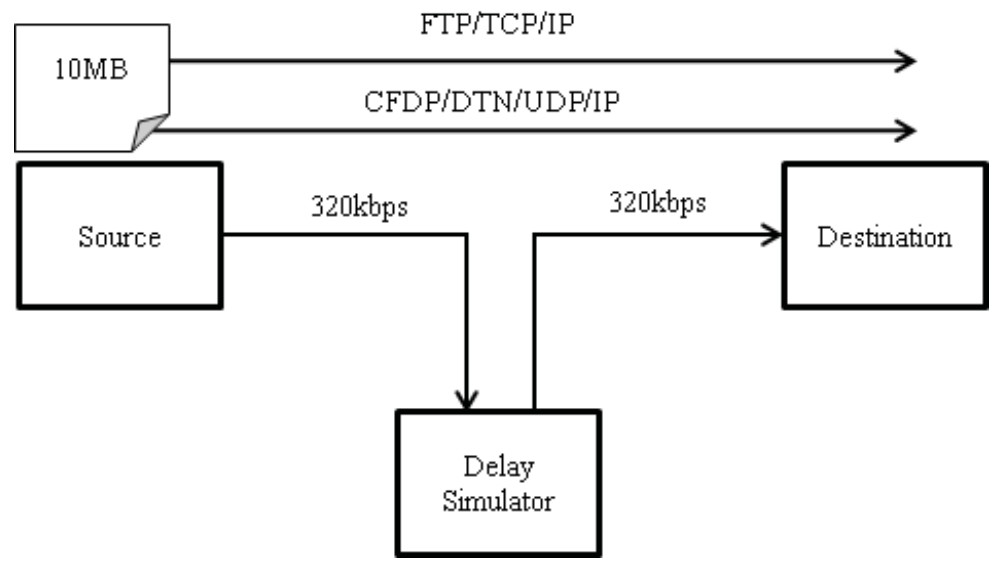

Figure 17. The configuration for feasibility study for comparison of delay

To impress delay, we used delay simulator. Herein, we used CFDP (CCSDS File Delivery Protocol) for DTN, and FTP for TCP/IP as file transfer application. Figure 18 illustrates the results of comparison.

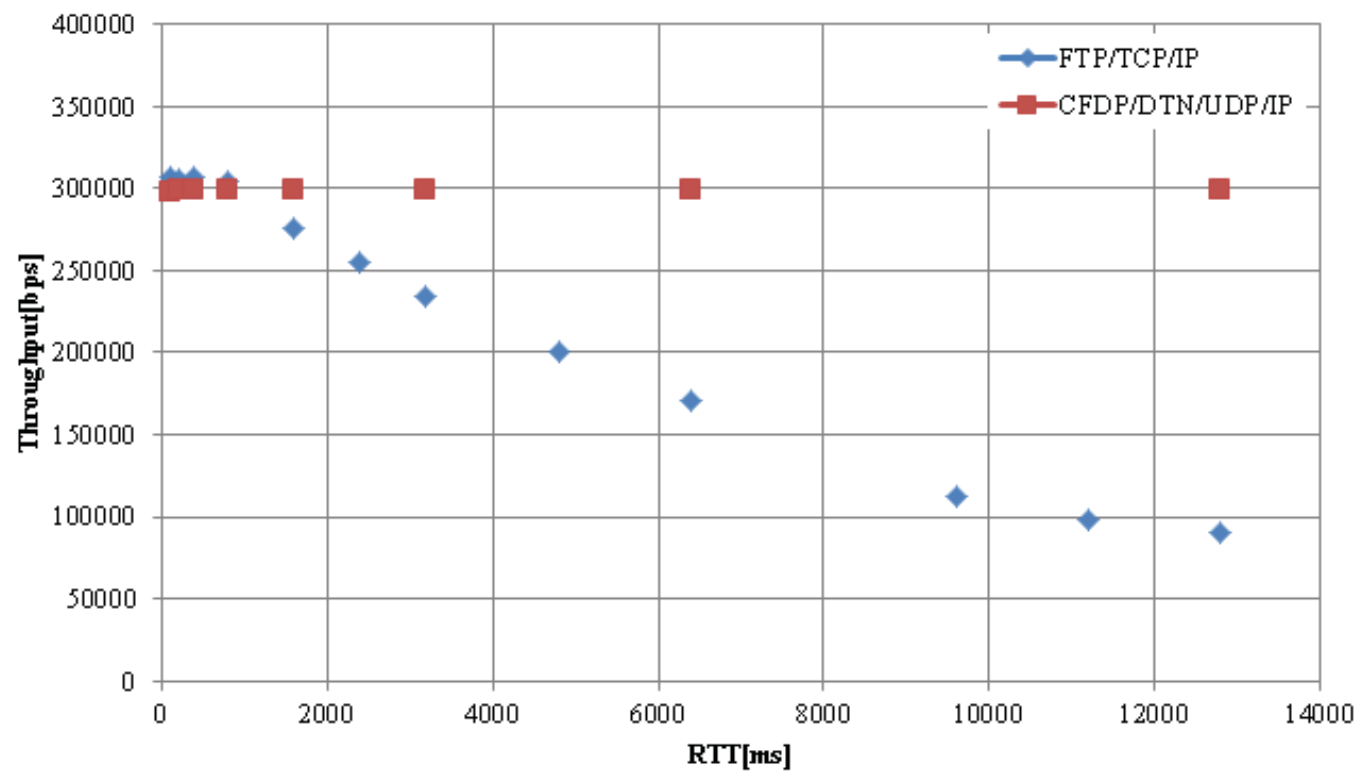

Figure 18. The result for feasibility study for comparison of delay

Our experience shows that a value of Timer_Initial_Value(T1_initial) [10] is around 10sec in case of even LEO spacecraft operation. That value is considered to include all of propagation delay and processing delay through endto-end space link protocol path. 
If you look at the Figure 18 , you will see that DTN maintained the throughput, and FTP dropped to a lower value than DTN's around $2 \mathrm{sec}$. As the result of this feasibility study, we concluded that it was reasonable to use DTN as a kind of SSI protocol from standpoint of delay tolerance at space segment.

\section{B. A proposal for concept of CGR from standpoint of operation}

The second point to be discussed was a proposal for an additional concept for CGR. In our testing, we used that a CGR implementation in ION. The calculation of this implementation was for all of contacts in topology.

The Global Exploration Roadmap (GER) in the International Space Exploration Coordination Group (ISECG) (www.globalspaceexploration.org/) is discussing sustainable human missions to the Mars. In there, we could image that various regional network existed such as Mars surface, relay communications, and so on. This means that the scalability of entire topology is up, and it is difficult for us to recognize all contacts of assets across region.

Therefore, we proposed an additional following concept for CGR based on our testing and the GER.

1) For calculation of routing, not only all of contacts for all assets, but also all of contacts within a regional network

2) In case of across regional network, gateway nodes were set.

3) Communication across region network was only communicated by gateway nodes.

4) In gateway node, difference character of each region network was considered such as protocol and device.

Figure 19 illustrates an example conceptual diagram for our proposed concept.

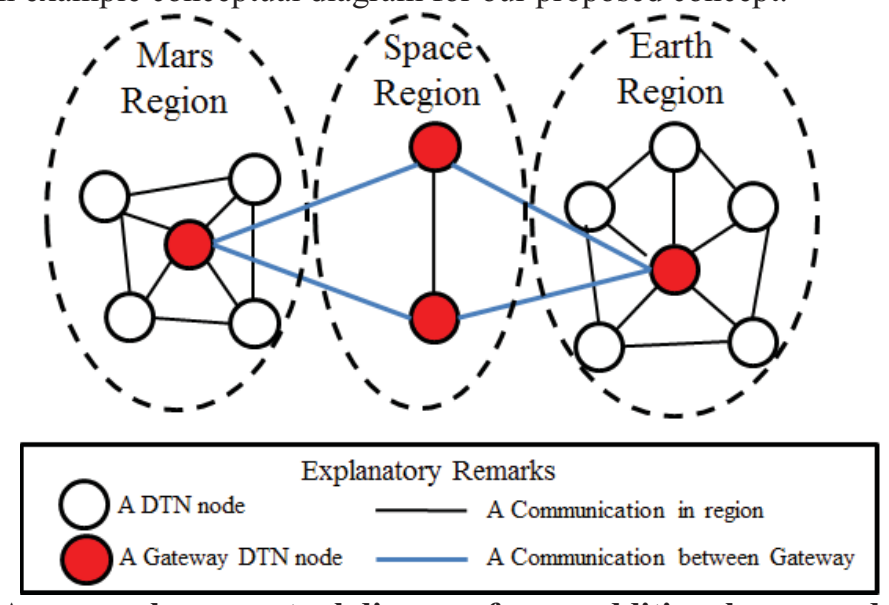

Figure 19. An example conceptual diagram for an additional proposed concept of CGR

\section{Conclusion}

In this paper, the authors performed DRTS testing to confirm a feasibility of future DTN usage for higher band space communications using DRTS's actual space link. We specifically focused on feasibility from the standpoint of delay and disruption included in simulated rain attenuation and long haul delay. From the testing results, we concluded that DTN has feasibility on an actual space link, even if there was long haul delay and rain attenuation.

In addition, we discussed that the DTN certainly had an advantage for SSI protocol over commodity internet, and we proposed an additional concept for CGR towards using future space exploration.

\section{Acknowledgement}

First of all, the authors dedicate this presentation to the honor of Dr. Adrian Hooke, who had paved our way into the future realization of interplanetary network and realization of JAXA and NASA DTN test activities with his intelligence and passion for the new technology. The authors would like to thank Dr. Keith Scott, Mr. Scott Burleigh, Mr. Shea Williams, and Dr. Kevin Gifford for technical observations and consultation. Also the authors would like to thank Mr. Heinrich Tsuchiya, Mr. Tadakatsu Abe, Mr. Katsuji Miyashita, and DRTS operators for technical support for our performing testing. 


\section{References}

1 " Rationale, Scenarios, and Requirements for DTN in Space ", CCSDS 734.0-G-1

2 "Solar System Internetwork architecture", CCSDS 730.G-0 (to be published)

${ }^{3}$ K. Scott, et. al "Bundle Protocol Specification", RFC 5050

${ }^{4}$ M.Ramadas, et.al "Licklider Transmission Protocol - Specification", RFC 5326

${ }^{5}$ S. Burleigh "draft-burleigh-dtnrg-cgr-00", draft-burleigh-dtnrg-cgr-00

${ }^{6}$ H.Uejima, et. al "DRTS Space Network Experiment System Overview “, $25^{\text {th }}$ AIAA International Communications Satellite Systems Conferences

7 " AOS Space Data Link Protocol “, CCSDS 732.0-B-2

8 " Encapsulation Service“, CCSDS 133.1-B-2

9 Kiyohisa Suzuki, et. al "A study for application of DTN under intermittently disrupted environment “, 2013 SpaceOps Workshop

10 "Communications Operation Procedure-1“, CCSDS 232.1-B-2 